Página inicial: 305 - Página final: 314

Tipo de artículo: de investigación

\title{
¿Qué está pasando con el agua en el Valle de Aburrá (Medellin-Colombia)?.
}

What is happening with the water in the el Aburra Valley?.

Recibido: Julio de 2015 Revisado: Octubre de $2015 \quad$ Aceptado: Noviembre 20 de 2015

Por: Alfonso Insuasty Rodriguez. ${ }^{1}$

\section{Resumen.}

El presente artículo, derivado de investigación, presenta un primer avance de algunas preguntas que han surgido respecto del abastecimiento sostenible y real de agua para Medellín, su calidad y los negocios que circulan en torno a este preciado recurso, presenta este texto, algunos hallazgos que nos posibilitan generar algunas líneas de análisis, con el fin de aportar en el necesario debate público, al respecto.

\section{Palabras clave.}

Agua, calidad del agua, abastecimiento, sostenibilidad, negocios.

\begin{abstract}
.
This article, derived from a piece of research, presents a first advance of questions, which have arisen with respect to the real and sustainable supply of water for Medellin, its quality and the businesses that circulate around this precious resource. This text presents some findings, which enable us to generate a number of lines of analysis, in order to contribute in the necessary public debate, in this regard.
\end{abstract}

\section{Key words.}

Water, Quality of water, Supply, Sustainability; and Businesses.

${ }^{1}$ Docente investigador, integrante del grupo de investigación para la transformación Kavilando y director del grupo GIDPAD de la Universidad de San Buenaventura, Editor de la revista El Ágora USB, y co-editor de la Revista Kavilando. Contacto: Alfonso.insuasty@usbmed.edu.co 


\section{Introducción.}

Los caudales del Cuenta Alta del Río Chico y del Río Grande abastecen el embalse Rio Grande II y es este líquido el que es aprovechado por la Empresas Pública de Medellín (EPM) para el abastecimiento del acueducto que surte a la ciudad de Medellín y algunos municipios del Valle de Aburra.

\section{EMBALSE Y CENTRALES DE ENERGÍA HIDROELÉCTRICA}

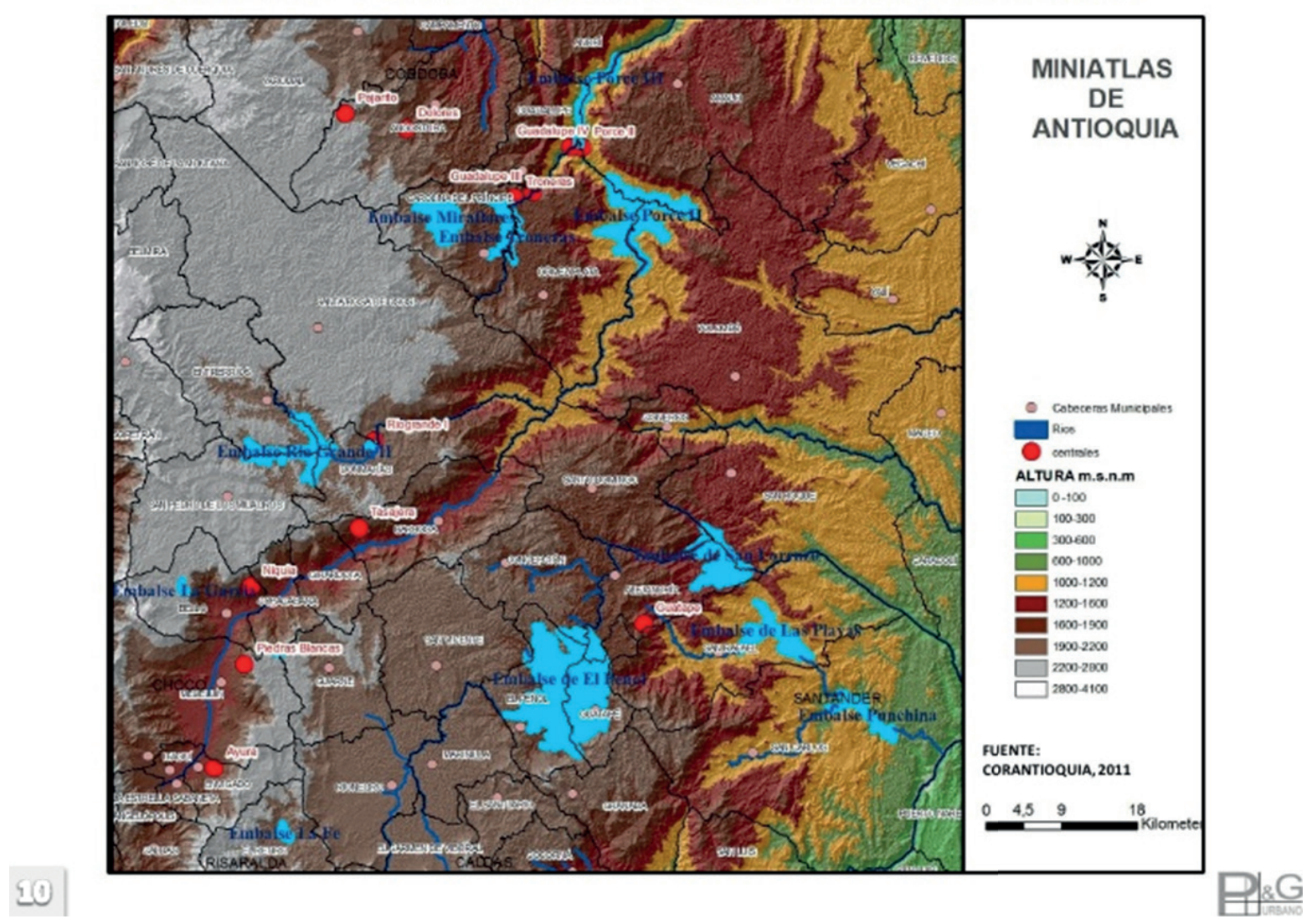

Imagen: http:/ /es.slideshare.net/CarlosHJaramilloA/3-miniatlas-antioquia-colombia-por-pg-urbano-carlos-hjaramillo-planificacin.

Otro importante servicio que genera el agua proveniente del Embalse Rio Grande II es el de la Energía, producida por las centrales hidroeléctricas de Niquia (Bella Ant.) y la Tasajera (Barbosa Ant.) las cuales proveen de energía tanto al Valle de Aburrá, como a otras zonas del Departamento y del país, a través del sistema de interconexión.

El Agua proveniente del Embalse Rio Grande II, luego de generar energía en la central hidroeléctrica La Tasajera, es también utilizada en el parque de las Aguas, (parque de recreación acuático) localizado en el municipio de Girardota (Antioquia) luego, es vertida dicha agua, en el Río Medellín para disminuir sus niveles de contaminación bajo en fenómeno de dilusión. 
Dicho embalse, que surte de Agua a Medellín, en los documentos oficiales, estima una vida útil de dicha estructura, hasta el año 2022.

\section{Metodologia.}

Para la elaboración de este artículo, derivado de investigación, se acudió a:

- Un rastreo bibliográfico sobre el tema.

- Rastreo de prensa que diera cuenta de la realidad carcelaria del País.

- Un análisis de los informes de Epm y estudios Universitarios sobre el tema.

- Rastreo jurídico de diferentes normas emitidas en torno al tema.

- Reflexiones derivadas del trabajo de campo que incluyó entrevistas, talleres, charlas informales y participación en actividades institucionales.

\section{Resultados.}

Varios estudios que se han realizado en torno al Embalse Río Grande II, el cuál abastece de Agua en porcentaje importante al Valle de Aburra dan cuenta de una serie de alertas que es necesario no solo identificar, sino hacer seguimiento.

Un estudio de agosto de 1978 titulado "Aprovechamiento múltiple del Rio grande", de Empresas Públicas de Medellín, sugería en su numeral IV, qué se debería hacer para el reordenamiento de la cuenca y la adquisición de tierras para proteger el agua y la calidad de la misma que va hacia el embalse, y el embalse mismo. Es así que se definen las áreas de construcción y las áreas críticas. Ambas, dice el documento, deben adquirirse por EPM, en virtud de proteger el ciclo del agua; definiéndose los instrumentos legales y los procedimientos para la adquisición de dichos bienes.

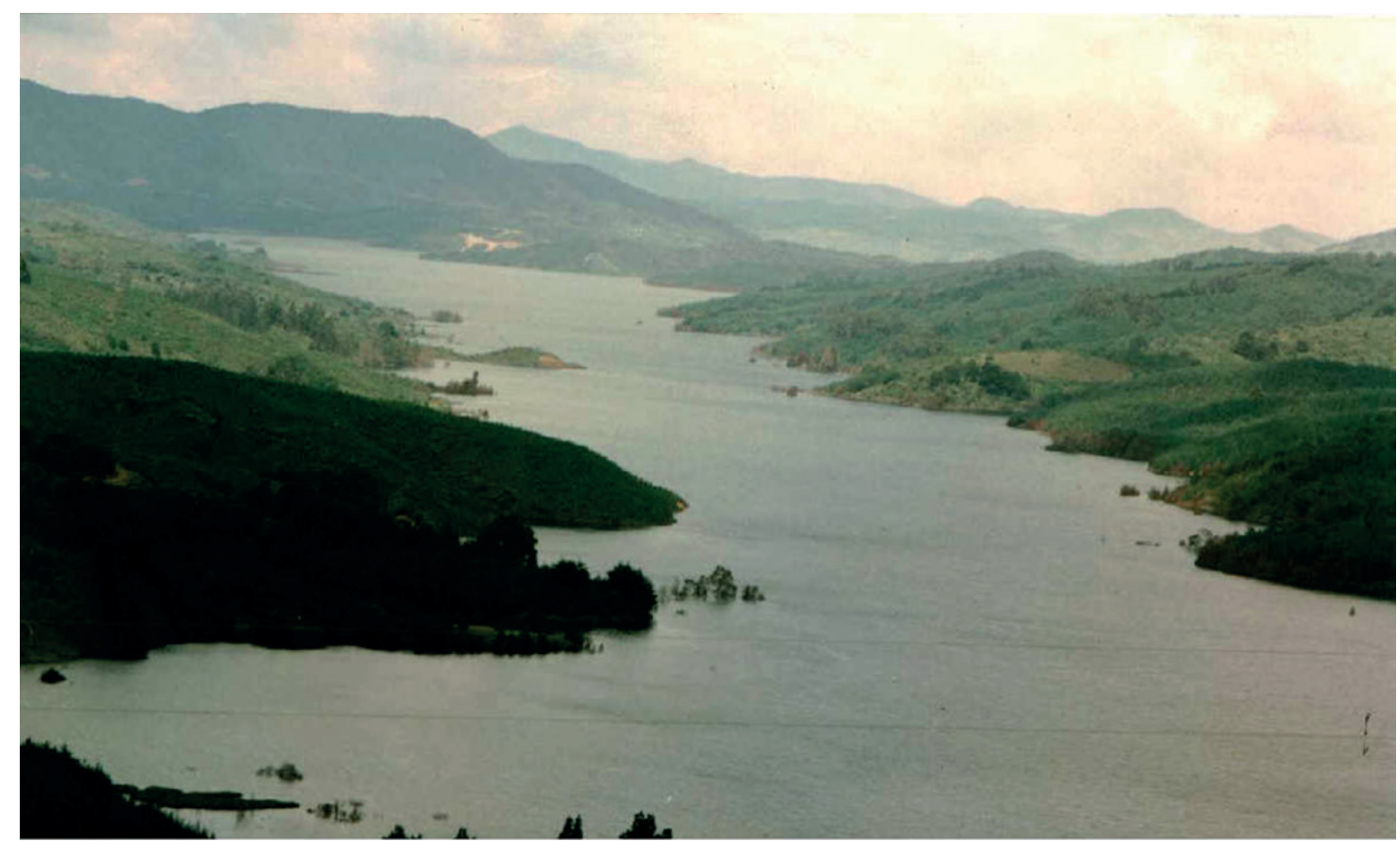

Embalse 1, Foto: entrerios-antioquia.gov.co 
Otro documento publicado en 1983, titulado "Aprovechamiento múltiple de Rio Grande, estudio de factibilidad de zonas críticas y sub-críticas de la cuenca de Rio Grande y tratamiento del área de embalse", en su volumen V llama la atención sobre tres problemas a la fecha del informe, respecto del informe incial "Aprovechamiento múltiple de Rio Grande": Erosión, manejo inadecuado del suelo y contaminación del agua.

Los tres estudios tienen en común:

Efectos causados por la minería, las prácticas agrícolas y pecuarias e infraestructura vial así como las condiciones
naturales del terreno aportan gran cantidad de sedimentos a la corriente de agua, la cual se expresa en su grado
de concentración.

"Otro factor son las curtimbres ubicadas en Entrerios, las aguas negras de la población ubicada en la cuenca del Rio Grande y Chico, todo contribuye a la eutroficación del agua". (EPM, 1983).

Este incumplimiento parcial frente a las recomendaciones, no solo aumenta el costo de la producción de energía sino el costo del tratamiento del agua, lo que se traduce en mayor riesgo y mayores tarifas en la factura mensual.

\section{El segundo informe presenta soluciones.}

Insiste en la compra de las tierras, medidas de mitigación, reforestación, entre otros. Estas medidas disminuirian el sedimento, mejorando la calidad del agua y los costos del tratamiento y producción de energía, así como la vida útil del embalse.

En el 2011 se publicó un Artículo Académico-investigativo sobre el tema, allí se aseguraba que:

"En éste punto, nos centraremos en un tema crítico y de repercusiones mayores para toda una comunidad, como lo es la degradación de las fuentes que aportan sus aguas al embalse Rio Grande II, con el consecuente deterioro del mismo, mediante procesos de eutroficación, pérdida de la capacidad hidráulica y alto costo de la potabilización de la fracción tomada para abastecimiento de la población y lo que es más grave aún, el riesgo que un proyecto de ésta magnitud no sea sostenible en el tiempo y pueda llegar a un punto de inviabilidad financiera, debido al alto costo de las soluciones de biorremediación" (Montoya Montoya \& Tamayo Cifuentes, 2011).

\section{Así mismo agrega:}

"La calidad de las aguas de los ríos Grande y Chico se ha venido afectando por impactos generados en la cuenca, asociados a las siguientes causas: Alteración del régimen hídrico con el desvio de cauces, desecación de humedales, extracción de materiales del río y alteración 
de la zonas de recarga y nacimientos por expansión de la frontera agrícola y ganadera. Uso inadecuado del suelo debido al sobrepastoreo y a la deforestación por las actividades mineras y agroindustriales, con el uso intensivo de agroquímicos. Inadecuada disposición de residuos sólidos y aguas residuales domésticas e industriales a nivel rural y urbano. Por todo lo anterior, el metabolismo global de estos ambientes limnéticos, así como el comportamiento espacial y temporal de las variables hidrológicas, químicas y biológicas que determinan su evolución y sostenibilidad, están profundamente afectados en su calidad y cantidad por las aguas provenientes de las cuencas aportantes a los embalses" (Montoya Montoya \& Tamayo Cifuentes, 2011). Agrega a los problemas del embalse el Buchon de agua.

En el 2013, se realizó una nueva investigación cuyas conclusiones ratifican lo dicho, y además advierte sobre la necesidad de pasar de las medidas de mitigación a la intervención y compra de las tierras para destinarlas a la reforestación para el cuidado de la cuenca, dicha investigación fue realizada por la Universidad Nacional con dineros de EPM y The Nature Conservancy (TNC) pero que cuenta con una cláusula de confidencialidad que por ahora imposibilita hacer referencia directa de dicho documento, lo que es lamentable pues se trata de un asunto público y de suma importancia por tratarse del agua que consumen los habitantes del Valle de Aburrá.

Laboratorios de la Bayer, vienen haciendo estudios sobre la calidad del agua en estas zonas en relación a los pesticidas que alli se vierten, no hemos logrado hacernos a los resultados de dichos estudios.

El 26 de diciembre de 2013, la secretaría Sección de Salud y Protección Social, Dirección de Factores de Riesgo, generó una circular dirigida a los secretarios de salud municipal, gerentes de ESE e IPS, Gerentes de entidades administradoras de Planes de Beneficios (EPS, ARS, ARP). Técnicos en área de salud (TAS), su asunto: Fortalecimiento de las acciones de prevención, promoción y vigilancia al contacto con algas de embalses (cianobacterias algas verde - azul) que producen cianotoxinas en donde establece las siguientes recomendaciones:

\begin{tabular}{|l|}
\hline No tome agua de los embalses \\
\hline No se bañe en los embalses \\
\hline No realice deporte acuático \\
\hline No realice actividades de pesca \\
\hline $\begin{array}{l}\text { Evite que los animales domésticos y el ganado la beban o entren en contacto con ella.(Secretaría seccional de } \\
\text { Salud y protección Social de Antioquia., 2013) }\end{array}$ \\
\hline
\end{tabular}

Vale decir que el problema de la detección de la cianobacteria es un fenómeno a desarrollar con mayor detenimiento, pues es un tema amplio y de cierta complejidad, solo relacionaremos lo que expresa la Dra Susana García, en su texto "Cianobacterias y cianotoxinas, impactos sobre la salud humana":

“... este florecimiento de algas ocurren de forma natural, pero son más frecuentes en aguas que han sufrido ciertas formas de interferencia humana, entre las cuales se destaca por su importancia, la eutrofización o enriquecimiento de las aguas con nutrientes, ya sea a partir de fuentes puntuales, tales como las descargas cloacales municipales, o de fuentes no puntuales como la agricultura o el endicamiento de los ríos, lo cual aumenta el tiempo de retención y de exposición del agua a la luz solar (...) éstas cianobacterias pueden generar 
serios problemas de salud (afecciones en la piel, gastrointestinales, malestar, intoxicación) en animales y humanos incluyendo la muerte. (García, sf).

El periódico el Mundo del 17 de enero de 2014, publicó un artículo titulado: Río Grande II recibe tratamiento. En esta publicación se corrobora lo dicho y se evidencia que dicha problemática persiste:

"Factores como el uso inadecuado del suelo, la deforestación, el sobrepastoreo, la utilización de pesticidas y fertilizantes, la inadecuada disposición de los residuos sólidos, las aguas residuales domésticas e industriales, y la alteración del régimen hídrico por el desvío de los cauces, han evidenciado un deterioro progresivo en la cuenca del Río Grande, causado directa e indirectamente por la intervención del hombre, afectando al embalse Río Grande 2". (Osorio Acosta, 2014).

El mismo texto publicado manifiesta que:

"El embalse Río Grande 2 funciona como una planta de tratamiento que recoge todas las aguas residuales de la parte alta de la cuenca del Río Grande.

Esta problemática que se viene presentando, puede generar a mediano plazo contaminación del agua y pérdida del volumen útil del embalse, a causa de la sedimentación por el arrastre del suelo y la tierra proveniente de la cuenca, afectando al 45\% del Valle de Aburrá que se abastece de agua potable gracias a esta fuente hídrica."

Es clave entender que este embalse genera energía y abastecerá de agua potable a gran parte del Valle de Aburrá a través de la planta Manantiales, Rio Grande y Rio Chico abastecen de Agua a dicho embalse el primero es importante porque tiene ecosistemas estratégicos como el páramo de Belmira (que tiene una solicitud de titulo minero en vilo) y "el sistema de humedales, además posee micro cuencas que son abastecedoras de agua para las poblaciones ubicadas en el altiplano norte, entre ellas San Pedro de los Milagros, Entrerríos, Don Matías y Santa Rosa de Osos, municipios importantes para el departamento por la producción de leche, los cultivos de papa, tomate y aguacate y su acelerado desarrollo agroindustrial”. (Osorio Acosta, 2014)

Preocupa que:

Ahora bien, la ley 99 del 93 dispuso en su artículo 111: "los departamentos y municipios dedicaran un porcentaje no inferior al $1 \%$ de sus ingresos corrientes para la adquisición y mantenimiento de las áreas de importancia estratégica para la conservación de recursos hídricos que surten de agua a los acueductos municipales, distritales y regionales, o para financiar esquemas de pago por servicios ambientales en dichas áreas."

Por un lado, la Contraloría General de la República, elevo una función de advertencia en torno a las múltiples debilidades técnicas, administrativas e institucionales de la gestión del recurso hídrico que impiden garantizar el agua como un bien público y derecho fundamental, humano y colectivo. (Contraloría General de la República, 2014) 
Del mismo modo TNC, WWF y Patrimonio Natural, advierten en sus informes, del incumplimiento que dichos municipios y Departamentos frente a la destinación de dicho recurso asignado y mandado por Ley. De tal forma que el 17 de Mayo de 2013, el Presidente de la República y el ministro de Medio Ambiente y desarrollo sostenible, expidieron el decreto 0953 con la Reglamentación del artículo 111 de la Ley 99 de 1993, modificado por el artículo 210 de la Ley 1450 de 2011 sobre el uso del 1\% previsto para compra, manejo de predios prioritarios, para el pago de servicios ambientales y asegurar el agua para consumo humano.

En medellín el concejo municipal, bajo pretexto de arriba expuesto, crea en octubre de 2013, una Alianza Público-Privada, dando origen a la Corporación Cuenca Verde, para el fin de gestionar "la consecución, administración, gestión, inversión, asignación y disposición de recursos financieros destinados a proteger, mantener, preservar los servicios ambientales, especialmente el agua, en las cuencas abastecedoras de los embalses que proveen de agua a los municipios del Valle de Aburra (principalmente Riogrande II y La Fe)". Dicha iniciativa se articula como parte de la alianza Latinoamericana de Fondos de Agua siendo el tercer fondo que se crea de este estilo en Colombia.

Esto preocupa lo que algunos llaman, pasos para la privatización de la gestión hídrica: sus entidades asociadas: Municipio de Medellín, EPM, Cornare, Grupo Nutresa, Postobón, Ärea Metropolitana del Valle de Aburrá, Coca Cola-FEMSA y la Alianza Latinoamericana de Fondos de Agua conformada por The Natura Conservancy (TNC), el Banco Interamericano del Desarrollo (BID) , la fundación FEMSA y el Fondo del Medio Ambiente Mundial (FMAM), igualmente cuenta con el apoyo del grupo Familia en calidad de Donante. (EPM, 2013)

Se crearán 32 fondos.

“ ¿Y cómo interviene el BID? Precisa que colabora a través de la Alianza Latinoamericana para los Fondos de Agua, que es un convenio entre la Fundación Femsa y el Fondo Mundial de Medio Ambiente, donde se constituyó una bolsa que apoya la creación de fondos de agua en Latinoamérica.

El objetivo es crear 32 fondos de agua en varias ciudades que se identificaron como las prioritarias por los posibles efectos del cambio climático a futuro, y esta es una de esas.

El vocero de The Nature Consevancy, Julio Cárcamo, considera que el nacimiento de Cuenca Verde asombra, incluso a una organización como la suya que promueve este tipo de proyectos a lo largo de Latinoamérica. Ya tiene al menos 15 y la meta son 30.

A Cárcamo le parece fundamental el alto nivel de empoderamiento, que los socios hablan por sus intereses y la independencia del concepto y necesidad del por qué es importante conservar el agua en el largo plazo.

El directivo de TNC también lo considera un excelente ejemplo de cómo el Gobierno y los actores privados se pueden unir por un objetivo común que no es egoísta, sino beneficioso para el ser humano". ( Saldarriaga L, 2013) 
A eteDe esta forma comenzará labores, después de 25 años de no ser aprobada una licencia para esta actividad, la primera empresa dedicada a la extracción de oro. Un antecedente que sin duda es el punto de partida para un nuevo discurso: la comunidad decide cómo y cuándo se hace minería en su territorio. "Las mineras tienen que entender que en todo lado no se puede extraer", reflexiona Ossa.

\section{Inician proyectos mineros.}

La multinacional Canadiense Red Eagle anunció que iniciará la construcción de una mina subterránea de oro en agosto de 2015 y que producirá su primera carga en 2017.

"La mina San Ramón producirá cerca de 400 mil onzas de oro entre 2017 y 2025, y 270 empleos directos -en la fase de construcción y de explotación- y 500 indirectos. "No estamos generando falsas expectativas. Explicamos cuál es la mano de obra que podemos generar. No queremos que las personas dejen el campo, por eso máximo habrá una persona por familia para que los otros no descuiden las actividades lecheras o ganaderas. No se puede perder la vocación agrícola", dijo Thyana Álvarez, directora de Responsabilidad Social de Red Eagle.

Según el director de la Unidad Municipal de Asistencia Técnica Agropecuaria (Umata), Nicolás Ossa, "esta zona es importante por la conservación de las aguas, pues abastecen a Medellín y el Valle de Aburrá. Casi un 50\% de las que se consumen son producidas en el norte y más del $60 \%$ de la electricidad que se usa allí se hace con estos recursos".

Lo que preocupa a esta compañia no es el medio ambiente, sino la caída de los precios internacionales". (Guesguán Serpa, 2015)

Por datos recogidos a través de derechos de petición, se tiene noticia de las múltiples solicitudes para la actividad extractiva minera en esta zona estratégica para la producción y conservación del agua.

\section{Conclusiones.}

"La problemática que viene siendo estudiada por EPM desde los años 90, ha generado la contaminación química, microbiológica y de materia orgánica del embalse; así como la disminución de la oferta hídrica en cuanto a la calidad y la cantidad del agua. Igualmente la torrencialidad por el aumento de los caudales y la erosión por las lluvias, han debilitado las orillas de los ríos provocando deslizamientos y obstrucción de las vías" (Osorio Acosta, 2014).

El agua, su calidad, el abastecimiento, la salud, el bienestar es lo que están en juego en esta larga historia de constantes informes, estudios, recomendaciones.

Las políticas trazadas a nivel nacional no se están cumpliendo o se implementan de manera amañada favoreciendo negocios privados.

La política económica extractiva se consolida aún por encima de la conservación estratégica del medio ambiente, aún por encima de la mínima provisión del agua para uso humano. 
La población en general está desinformada y ausente de los profundos debates que tocan no solo su presente sino pro sobre todo, su futuro.

Debido al gran desconocimiento de la ciudadanía, esta problemática no se percibe como un problema real, más allá de cerrar la llave del lavamanos como una superficial campaña que no toca los problemas estructurales.

La afectación directa también a las tarifas su costo es también una consecuencia de dichas fallas y su desconocimiento evita una movilización social contundente y oportuna.

"El discurso sobre la naturaleza, se ha disparado y se hace necesario hacernos propuestas, ¿qué es lo que significan?, ¿Acaso existe la posibilidad de cambiar el estado actual con respecto a la naturaleza, lo ecológico desde una nueva mentalidad individual, social, politica y religiosa respetuosa de la naturaleza tanto humana como fisica? ¿podemos hablar de respeto de la naturaleza en el marco del actual sistema capitalista, así él se presente con cara amable y verde?

Los temas actuales en materia de desarrollo humano tocan de una u otra manera la realidad ecológica, constituyéndose hoy, en un punto de referencia importante para hablar de cualquier discurso ya sea en el ámbito de lo político, lo religioso, lo económico, lo social, lo ético, entre otros.

Es necesario abrir la escucha, construir nuevas maneras de comprender nuestro entorno, de construir sentidos, no un sentido, sino en plural, mundos posibles; en ésta lógica, las propuestas desde el pensamiento ancestral-latinoamericano, son bien importantes, y se debe avanzar en ganar espacios de reconocimiento y de diálogo con múltiples propuestas que buscan rutas para construir mundos posibles que superen las maneras de relacionarnos depredadoras, acumuladoras, egocéntricas, individualistas” (Herrera Ospina \& Insuasty Rodriguez, 2015)

Urge abrir el debate, hacer pública la información, preocupa también la alianza "más privada que pública", que se articula para "cuidar el agua", sabemos que el sistema de mercado global no camina sin amarres convenientes, ¿Qué sigue? ¿Qué debemos hacer?.

\section{Referencias Bibliográficas.}

Saldarriaga L, L. (24 de Octubre de 2013). Fondo del Agua protegerá La Fe y Riogrande II. Obtenido de El Colombiano: http://www.elcolombiano.com/fondo_del_agua_protegera_ la_fe_y_riogrande_ii-HYEC_266661

Contraloría General de la República. (23 de 07 de 2014). Debilidades técnicas, administrativas e institucionales de la gestión del recurso hidrico que impiden garantizar el agua como un bien público y derecho fundamental, humano y colectivo. Obtenido de http://www.contraloriagen.gov.co/: http://www.contraloriagen.gov.co/ documents / 10136/182814564/FA+2014EE0123916MinAmbienteRecursoHidrico.pdf/ c0161cbc-d6d3-455e-a3f2-3633286131da 
EPM. (1978). Aprovechamiento multiple de Rio Grande. Medellín: Empresas Públicas de Medellín.

EPM. (1983). Aprovechamiento múltiple de Rio Grande. Medellín: Empresas Públicas de Medellin.

EPM. (2009). Gestión integral de embalses en EPM. Obtenido de www.epm.com.co: http:/ www.epm.com.co

EPM. (24 de Octubre de 2013). Corporación Cuenca Verde. Obtenido de www.epm.com.co: http://www.epm.com.co/site/Portals/0/documentos/noticias/Cuenca_Verde.pdf

García, S. I. (s.f.). Cianobacterias y cianotoxinas, impactos sobre la salud humana. Obtenido de www.ataonline.org: http://www.ataonline.org.ar/bibliotecavirtual/documentos_ utilies/Cianobacterias_y_Cianotoxinas.pdf

Guesguán Serpa, O. (18 de Julio de 2015). Santa Rosa de Osos brilla como el oro. Obtenido de El Espectador: http://www.elespectador.com/noticias/economia/santa-rosa-deosos-brilla-el-oro-articulo-573217

Herrera Ospina, J. d., \& Insuasty Rodriguez, A. (2015). Diversas concepciones en torno a la naturaleza como sujeto político. El Agora USB, 15(2), 537-555.

Insuasty Rodriguez, A. (18 de Abril de 2014). ¿qué está pasando con el Agua en el Valle de Aburrá? Obtenido de www.kavilando.org: http://kavilando.org/index.php/8editorial/2726-que-esta-pasando-con-el-agua-en-el-valle-de-aburra

Montoya Montoya, J. A., \& Tamayo Cifuentes, L. F. (2011). Embalse Rio Grande II. Antioquia. Obtenido de www.ecosistemastropicalesucn.blogspot.com.co: http:// ecosistemastropicalesucn.blogspot.com.co/2011/03/embalse-riogrande-ii-antioquia. html

Osorio Acosta, M. F. (14 de Enero de 2014). Rio Grande II recibe tratamiento. Obtenido de www.elmundo.com: http://www.elmundo.com/portal/pagina.general.impresion. php?idx $=161603$

Secretaría seccional de Salud y protección Social de Antioquia. (26 de Diciembre de 2013). Fortelacimiento de las acciones de prevención, promoción y vigilancia al contacto con algas de embalses (cianobacterias). Obtenido de www.dssa.gov.co: https://www.dssa.gov. co/index.php/documentos-de-interes / normatividad/circulares / circulares-2013/27circular-000494-de-26-diciembre-de-2013/file 